\title{
Systemic Therapy for Soft Tissue Sarcoma: Proposals for the Optimal Use of Pazopanib, Trabectedin, and Eribulin
}

\author{
Akira Kawai · Kan Yonemori · Shunji Takahashi · Nobuhito Araki • \\ Takafumi Ueda
}

Received: March 31, 2017 / Published online: May 25, 2017

(C) The Author(s) 2017. This article is an open access publication

\section{ABSTRACT}

Soft tissue sarcoma (STS) is a rare tumor with more than 50 histologic subtypes. Although treatment outcomes for patients with STS have improved greatly over the past few decades owing to the adoption of a multidisciplinary approach, patients with advanced disease have a poor prognosis. The development of

Enhanced content To view enhanced content for this article go to http://www.medengine.com/Redeem/6A48 F060509C3B10.

\footnotetext{
A. Kawai $(\bowtie)$

Department of Musculoskeletal Oncology and Rehabilitation Medicine, National Cancer Center Hospital, Tokyo, Japan

e-mail: akawai@ncc.go.jp

K. Yonemori

Department of Breast and Medical Oncology,

National Cancer Center Hospital, Tokyo, Japan

S. Takahashi

Department of Medical Oncology, The Cancer Institute Hospital of Japanese Foundation for

Cancer Research, Tokyo, Japan

N. Araki

Department of Orthopaedic Surgery, Osaka

Prefectural Hospital Organization, Osaka

International Cancer Institute, Osaka, Japan

T. Ueda

Department of Orthopaedic Surgery, National Hospital Organization, Osaka National Hospital, Osaka, Japan
}

anticancer drugs has been directed toward improving overall survival (OS). Doxorubicin monotherapy is currently the only standard option for the first-line treatment of STS. However, there is no standard therapy for second-line and later treatment at present. Since 2012, three anticancer drugs-pazopanib, trabectedin, and eribulin-have been approved in Japan for the second-line or later treatment of patients with advanced STS of any histologic subtype. However, the chemosensitivity of STS to each of these drugs varies by histologic subtype and their safety profiles differ; thus, histologic subtype and patient characteristics must be considered when determining optimal treatment. In this article, we review data from clinical studies related to the efficacy of all three drugs, including their effect on OS, and propose optimal treatment strategies for advanced STS by histologic subtype. In addition, with regard to the safety profiles, we highlight the key issues to be considered when selecting patients for treatment with pazopanib, trabectedin, or eribulin and ensuring their appropriate use, based on our combined clinical experience as specialists in the treatment of patients with STS. The proposed treatment strategies as well as treatment precautions based on clinical experience would benefit patients by maximizing the therapeutic effects and enhancing the proper use of these drugs.

Funding: Eisai Co., Ltd. 
Keywords: Eribulin; Evidence-based medicine; Japan; Leiomyosarcoma; Liposarcoma; Optimal use; Pazopanib; Proper use; Soft tissue sarcoma; Trabectedin

\section{INTRODUCTION}

\section{Epidemiology of Soft Tissue Sarcoma}

Soft tissue sarcoma (STS) is a rare tumor (less than $1 \%$ of all tumors) with an age-adjusted annual incidence of 2.8 per 100,000 people (World Health Organization world standard population) [1] and an age-adjusted incidence rate of 2.40 per 100,000 person-years (based on US data) [2]. In the USA, there are expected to be 12,390 new cases of STS in 2017, resulting in 4990 deaths [3]. In Japan, 1769 cases of STS were registered in 2015, and a 5-year overall survival (OS) of about 78\% was reported [4] (these data were collected by orthopedic surgeons only, which may explain differences from data from other sources). STS can arise in almost any anatomic site, including the extremities (60\% of cases), thorax, abdomen, retroperitoneum, and head and neck region [5]. More than 50 histologic subtypes of STS, each with different treatment response and prognosis, have been identified and are defined in accordance with the World Health Organization classification $[6,7]$. The wide range of histologic subtypes of STS, and its occurrence at various sites, means that affected patients are treated by various clinical departments; thus, it is difficult to obtain a general overview of STS.

Treatment outcomes for patients with STS have improved greatly over the past few decades. This is attributable to the adoption of a multidisciplinary approach to management of the disease, including surgery, radiotherapy, and systemic therapy. However, the 5-year OS of STS patients taken as a whole remains about $50 \%$, which is far from satisfactory $[5,8]$. This is because, although a 5-year OS of approximately $90 \%$ is achieved in patients with early-stage STS, cases of unresectable tumor (which cannot be cured by surgical removal), including those with locally advanced or metastatic disease at diagnosis, have a particularly poor prognosis; for these cases, the 5-year survival rate is only 20-50\% [5]. Therefore, treatment with anticancer drugs has been directed toward improving OS.

\section{Current Systemic Therapy for Advanced STS}

In cases of unresectable and/or metastatic STS, i.e., advanced STS, anticancer drugs used in first-line treatment include doxorubicin, ifosfamide, and dacarbazine; these are used alone or in combination to prolong OS [9-14]. Several clinical studies have shown that combination therapy with doxorubicin and other anticancer drugs can improve treatment response and progression-free survival (PFS), but there is no significant difference from doxorubicin monotherapy in OS [11-14]. Moreover, the use of combination therapy may be limited by adverse effects and reduced feasibility; thus, doxorubicin monotherapy is currently the standard first-line treatment. Nevertheless, the European Society for Medical Oncology guidelines suggest that combination therapy including anthracyclines may be the treatment of choice in patients with good performance status [15]. Therefore, a combination of anticancer drugs to achieve a good tumor response may be considered in patients with increased symptoms and decreased quality of life due to tumor growth.

Against this background, three new anticancer drugs-pazopanib, trabectedin, and eribulin-have been approved in Japan since 2012 as second-line or later treatment options for patients with advanced STS.

\section{Objectives}

Because STS is a rare tumor, can occur throughout the body, and is treated by various clinical departments, it is difficult for physicians to gain sufficient clinical experience in its treatment. Moreover, few comprehensive reviews are available to guide them in the use of pazopanib, trabectedin, and eribulin. Therefore, in this article we aim to provide information on each drug's profile, appropriate target patients, 
and proper use of the drugs, which will eventually lead to benefits for STS patients and for physicians treating STS patients. We also aim to highlight the key issues regarding treatment strategies and proper use of the three anticancer drugs for the treatment of advanced STS, based on our review of research conducted in accordance with evidence-based medicine and our combined clinical experience as sarcoma specialists and oncologists.

\section{METHODS}

To achieve our stated objectives, we reviewed and discussed data from clinical studies of pazopanib, trabectedin, and eribulin. When selecting prospective clinical studies, the level of evidence was considered to avoid positional bias and maintain objective impartiality. In accordance with our review criteria, from reports published up to December 2016 of clinical studies of the three drugs that enrolled patients with advanced STS who had received previous treatment, we used data from phase III studies (highest evidence level), subgroup analyses of phase II and III studies according to histologic subtype, and prospective clinical studies in Japanese. This article is based on previously conducted studies and does not involve any new studies of human or animal subjects performed by any of the authors.

In the first section, "Review of Key Evidence", we assess the efficacy of each drug by using data from the main analyses of the relevant phase III studies, which have the highest level of clinical evidence. In the second section, "Review of Histologic Subtypes", we evaluate the efficacy of each drug for different histologic subtypes of STS. Because the numbers of patients with each histologic subtype were limited and we aim to maximize the data collected, we reviewed the relevant data from subgroup analyses of both phase II and phase III studies. In the third section, "Safety Considerations", we review the safety of each drug. To include prospective information on safety of each drug in Japanese patients, we used safety data from the phase III studies, subgroup analyses of data from Japanese patients included in the international phase III study, and two phase II studies conducted in Japan. In addition, we discuss the safety issues to be considered when selecting patients for treatment with pazopanib, trabectedin, and eribulin, based on our combined clinical experience.

\section{RESULTS}

\section{Review of Key Evidence}

We reviewed data from the main analyses of the phase III studies of the three drugs [16-18]; their efficacy is summarized in Table 1 . The patient characteristics varied between the studies. Regarding the main histologic subtype, the proportions of patients with liposarcoma, leiomyosarcoma, and other histologic subtypes were $0 \%, 44.7 \%$, and $55.3 \%$, respectively, in the phase III study of pazopanib; $27 \%, 73 \%$, and $0 \%$, respectively, in the phase III study of trabectedin; and $33.8 \%, 65.7 \%$, and less than $1 \%$, respectively, in the phase III study of eribulin. Regarding the number of previous chemotherapy regimens for advanced STS defined in the inclusion criteria, for pazopanib the requirement was at least one regimen containing anthracycline and a maximum of four previous lines of systemic therapy for metastatic disease (no more than two lines of combination regimens); for trabectedin, the requirement was at least either a combination of an anthracycline and ifosfamide or an anthracycline plus one or more additional cytotoxic chemotherapy regimen(s); for eribulin, the requirement was at least two standard systemic regimens including an anthracycline. All studies included patients who had received the standard treatment for STS, namely an anthracycline (e.g., doxorubicin)-containing regimen, and the median number of previous chemotherapy regimens in the three studies was two.

\section{Impact of Prolonged Overall Survival in Cancer Therapy}

The aims of treatment for advanced STS are to reduce symptoms and prolong survival. 
Table 1 Efficacy reported in phase III studies

\begin{tabular}{|c|c|c|c|c|c|c|c|}
\hline \multicolumn{2}{|l|}{ Treatment } & \multicolumn{2}{|l|}{ Pazopanib } & \multicolumn{2}{|l|}{ Trabectedin } & \multicolumn{2}{|l|}{ Eribulin } \\
\hline \multicolumn{2}{|l|}{ Study } & \multicolumn{2}{|l|}{ PALETTE } & \multicolumn{2}{|c|}{ ET743-SAR-3007 } & \multicolumn{2}{|c|}{ E7389-G000-309 } \\
\hline \multicolumn{2}{|l|}{$n$} & \multicolumn{2}{|l|}{369} & \multicolumn{2}{|l|}{518} & \multicolumn{2}{|l|}{452} \\
\hline \multicolumn{2}{|c|}{ Previous chemotherapy } & \multicolumn{2}{|c|}{$\begin{array}{l}\text { At least one regimen containing } \\
\text { anthracycline and a maximum of } \\
\text { four previous lines of systemic } \\
\text { therapy for metastatic disease (no } \\
\text { more than two lines of } \\
\text { combination regimens) }\end{array}$} & \multicolumn{2}{|c|}{$\begin{array}{l}\text { At least either a combination of an } \\
\text { anthracycline and ifosfamide or an } \\
\text { anthracycline plus one or more } \\
\text { additional cytotoxic chemotherapy } \\
\text { regimen(s) }\end{array}$} & \multicolumn{2}{|c|}{$\begin{array}{l}\text { At least two standard systemic regimens } \\
\text { including an anthracycline }\end{array}$} \\
\hline \multicolumn{2}{|c|}{$\begin{array}{l}\text { Median numbers of previous } \\
\text { chemotherapy regimen }\end{array}$} & \multicolumn{2}{|l|}{2} & \multicolumn{2}{|l|}{2} & \multicolumn{2}{|l|}{2} \\
\hline $\begin{array}{l}\text { Experimental } \\
\operatorname{arm}(n)\end{array}$ & $\begin{array}{l}\text { Comparator } \\
\operatorname{arm}(n)\end{array}$ & $\begin{array}{l}\text { Pazopanib } \\
(246)\end{array}$ & Placebo (123) & $\begin{array}{l}\text { Trabectedin } \\
(345)\end{array}$ & Dacarbazine (173) & Eribulin (228) & Dacarbazine (224) \\
\hline \multirow{2}{*}{\multicolumn{2}{|c|}{ Primary end point }} & \multicolumn{2}{|l|}{ PFS } & \multicolumn{2}{|l|}{ OS } & \multicolumn{2}{|l|}{ OS } \\
\hline & & \multicolumn{2}{|l|}{ Met } & \multicolumn{2}{|l|}{ Unmet } & \multicolumn{2}{|l|}{ Met } \\
\hline \multirow{2}{*}{\multicolumn{2}{|c|}{ Secondary end point }} & \multicolumn{2}{|l|}{ OS } & \multicolumn{2}{|l|}{ PFS } & \multicolumn{2}{|l|}{ PFS } \\
\hline & & \multicolumn{2}{|l|}{ Unmet } & \multicolumn{2}{|l|}{ Met } & \multicolumn{2}{|l|}{ Unmet } \\
\hline \multicolumn{2}{|l|}{ Histology } & \multicolumn{2}{|c|}{ Non-liposarcoma } & \multicolumn{2}{|c|}{ Liposarcoma, leiomyosarcoma } & Liposarcoma, 1 & yosarcoma \\
\hline & Median (months) & 12.5 & 10.7 & 12.4 & 12.9 & 13.5 & 11.5 \\
\hline OS & $\mathrm{HR}(95 \% \mathrm{CI})$ & $0.86(0.67-$ & & 0.87 & & $0.77(0.62-0.9$ & \\
\hline & $P$ value & $0.25^{\mathrm{a}}$ & & $0.37^{\mathrm{b}}$ & & $0.0169^{\mathrm{c}}$ & \\
\hline & Median (months) & 4.6 & 1.6 & 4.2 & 1.5 & 2.6 & 2.6 \\
\hline PFS & HR $(95 \% \mathrm{CI})$ & $0.31(0.24$ & & $0.55(0.44-0$ & & $0.88(0.71-1.0$ & \\
\hline & $P$ value & $<0.0001^{\mathrm{a}}$ & & $<0.001^{\mathrm{b}}$ & & $0.23^{c}$ & \\
\hline
\end{tabular}

$C I$ confidence interval, $H R$ hazard ratio, $O S$ overall survival, $P F S$ progression-free survival

a By two-sided stratified Wald test

b By unstratified log-rank test

c By two-sided stratified log-rank test

Therefore, improvements in OS have a large clinical impact, particularly in cases of recurrent STS with a poor prognosis. Recent phase III studies for cancer therapy tend to have OS as the primary end point. In a phase III study designed to evaluate the new drug olaratumab as a first-line treatment option for advanced STS, the primary end point is OS [19].

\section{Pazopanib}

The PALETTE (PAzopanib expLorEd in sofT-Tissue sarcoma-a phasE III study) trial was an international, multicenter, randomized, double-blind, placebo-controlled phase III study designed to evaluate the clinical benefits of pazopanib in patients with advanced STS [16]. Patients with metastatic STS progressing despite 
previous standard chemotherapy $(n=369)$ were randomized to either the pazopanib arm (800 mg orally once daily; $n=246$ ) or the placebo arm $(n=123)$ of the study in a ratio of $2: 1$. The primary end point was PFS, and the secondary end points included OS. Median PFS was 4.6 months in the pazopanib arm and 1.6 months in the placebo arm, showing a significant improvement in PFS in the pazopanib arm (hazard ratio, HR $0.31 ; 95 \%$ confidence interval, CI $0.24-0.40 ; P<0.0001)$; however, there was no significant improvement in OS (HR 0.86; 95\% CI 0.67-1.11; $P=0.25$ ) (Table 1). Patients with liposarcoma were excluded from this phase III study, because the phase II study showed insufficient evidence for the efficacy of pazopanib in the treatment of liposarcoma [20].

\section{Trabectedin}

The ET743-SAR-3007 trial was a multicenter, randomized, open-label, active-controlled, parallel-group, phase III study designed to evaluate the clinical benefits of trabectedin in patients with advanced STS [17]. Patients with locally advanced or metastatic STS after previous therapy with an anthracycline and at least one additional systemic regimen $(n=518)$ were randomized to either the trabectedin arm $(n=345)$ or the dacarbazine arm $(n=173)$ of the study in a ratio of $2: 1$. In the trabectedin arm, trabectedin $\left(1.5 \mathrm{mg} / \mathrm{m}^{2}\right)$ was administered as a 24-h intravenous infusion, whereas in the dacarbazine arm, dacarbazine $\left(1 \mathrm{~g} / \mathrm{m}^{2}\right)$ was administered by intravenous infusion over 20-120 min. In each arm, the study drug was administered on day 1 of every 21 -day cycle. OS was the primary end point, and PFS was a secondary end point. PFS was significantly improved in the trabectedin arm compared with the dacarbazine arm (median PFS, 4.2 versus 1.5 months; HR 0.55 ; 95\% CI 0.44-0.70; $P<0.001)$; however, interim analysis showed no improvement in OS, the primary end point (HR $0.87 ; P=0.37$ ) (Table 1 ). One reason for the non-significant result for OS may be the effect of the active comparator, dacarbazine [17].

It should be noted that the approved dose of trabectedin in Japan is different from that in the USA and Europe; the approved dose in Japan, $1.2 \mathrm{mg} / \mathrm{m}^{2}$, which was used in the
ET743-SAR-3007 phase III study, is lower than the $1.5 \mathrm{mg} / \mathrm{m}^{2}$ dose used in other countries. The approved dose in Japan is based on the results of a phase I study in Japanese advanced STS patients who had received treatment with at least one anthracycline-based regimen [21]. Dose-limiting toxicity was experienced by two of three patients who received trabectedin at a dose of $1.5 \mathrm{mg} / \mathrm{m}^{2}$; one had grade 3 increased creatine phosphokinase and grade 3 anorexia, and the other had grade 4 decreased platelet count.

\section{Eribulin}

The phase III E7389-G000-309 trial was conducted as a multicenter, open-label, active-controlled, parallel-group study to evaluate the clinical benefits of eribulin in patients with advanced STS [18]. Patients with locally recurrent, locally advanced, or metastatic STS who had received treatment with systemic regimens including an anthracycline $(n=452)$ were randomized to either the eribulin arm $(n=228)$ or the dacarbazine arm $(n=224)$ in a ratio of $1: 1$. In the eribulin arm, eribulin mesylate $\left(1.4 \mathrm{mg} / \mathrm{m}^{2}\right)$ was administered as an intravenous infusion over $2-5 \mathrm{~min}$ on days 1 and 8 of every 21-day cycle, whereas in the dacarbazine arm, dacarbazine $(850,1000$, or $1200 \mathrm{mg} / \mathrm{m}^{2}$ ) was administered as an intravenous infusion over $15-60 \mathrm{~min}$ on day 1 of every 21-day cycle. The primary end point was OS, and the secondary end points included PFS. OS was significantly improved in the eribulin arm compared with the dacarbazine arm (median OS, 13.5 versus 11.5 months; HR 0.77 ; 95\% CI $0.62-0.95 ; P=0.0169)$; however, there was no significant difference between the two arms in the secondary end point of PFS (HR $0.88 ; 95 \%$ CI 0.71-1.09; $P=0.23$ ) (Table 1 ).

\section{Review of Histologic Subtypes}

In the treatment of advanced STS, it is necessary to choose an anticancer drug that is effective against the specific histologic subtype. However, because STS is a rare tumor, it is difficult to prospectively accumulate clinical data from patients with different histologic subtypes to 
evaluate the efficacy of particular drugs against each subtype. Therefore, the findings of subgroup analyses by histologic subtype should be taken into account when deciding which drug to use in clinical practice. The chemosensitivity of each histologic subtype to anticancer drugs must also be considered. Therefore, in this section, we review subgroup analyses of data from the phase III studies [16-18] and phase II studies $[20,22]$ of pazopanib, trabectedin, and eribulin, and propose treatment strategies for advanced STS.

\section{Chemosensitivity of Different Histologic Subtypes of STS}

The chemosensitivity of each histologic subtype to anticancer drugs is described in UK guidelines for the management of STS [23] and in an article summarizing the opinions of international experts regarding patient-tailored management of STS [24]. On the basis of this information and our combined clinical experience, we examined the treatment for each histologic subtype, and list the primary drugs of choice and substitution drugs of choice in Table 2.

\section{Liposarcoma}

In the international multicenter phase III study of pazopanib [16], patients with liposarcoma were not included in the study. This is because in the phase II study [20], the primary end point, namely progression-free rate at 12 weeks $\left(\mathrm{PFR}_{12}\right.$ weeks $)$, was $26 \%$, which was lower than the $44 \%$ value for leiomyosarcoma, $49 \%$ for synovial sarcoma, and 39\% for other types of STS, as well as the benchmark value of $\mathrm{PFR}_{12}$. weeks (40\%); this last value was used as the minimum at which an anticancer drug can be considered effective as a second-line treatment for STS in phase II studies, based on information from a database of clinical studies including data from patients with various subtypes of STS [25]. Therefore, current evidence suggests that eribulin and trabectedin are preferable to pazopanib for the treatment of liposarcoma.

Subgroup analysis by histologic subtype, using data from a phase III study, has shown trabectedin to be effective in terms of improvement in PFS for patients with myxoid/ round-cell liposarcoma (HR $0.41 ; 95 \%$ CI $0.17-0.98)$ [17]. The effect of trabectedin on these subtypes was also shown in a randomized, phase II study in Japanese patients with translocation-related sarcoma (HR 0.03; 95\% CI $<0.01-0.22$ ) [22].

Subgroup analysis by histologic subtype, using data from a phase III study (the E7389-G000-309 trial), has shown eribulin to be effective in terms of improvement in OS for patients with liposarcoma (HR 0.51; 95\% CI 0.35-0.75) [18]. Therefore, eribulin can be expected to improve OS in patients with liposarcoma. Further analyses showed eribulin to be effective for patients with dedifferentiated liposarcoma (HR 0.45 ; 95\% CI $0.25-0.82$ ) and pleomorphic liposarcoma (HR 0.21; 95\% CI $0.06-0.76)[26]$.

On the basis of the above information, we propose the treatment strategy for liposarcoma shown in Fig. 1. Eribulin may be the optimal treatment option for liposarcoma, particularly dedifferentiated liposarcoma and pleomorphic liposarcoma after doxorubicin treatment. Trabectedin may be the first option for myxoid/ round-cell liposarcoma after doxorubicin treatment. Also, either eribulin or trabectedin can be used for each liposarcoma, depending on conditions such as safety issues and method of administration (for inpatients or outpatients).

\section{Leiomyosarcoma}

Patients with leiomyosarcoma made up the greatest proportion $(44.7 \%)$ of the study population in the international phase III study of pazopanib [16], in which the primary end point of PFS was met. Subgroup analysis by histologic subtype, as well as predictive analysis for histology subtype using Cox models, showed pazopanib to be effective for leiomyosarcoma in terms of PFS [16].

In the phase III study of trabectedin, patients with leiomyosarcoma made up the greatest proportion $(73 \%)$ of the study population, and the primary end point of OS was not met [17]; however, trabectedin treatment resulted in similar OS compared with dacarbazine (the active comparator). On the other hand, subgroup analysis of data for the secondary end point (PFS) showed an improvement with trabectedin compared with 
Table 2 Chemosensitivity of different histologic subtypes of soft tissue sarcoma and treatment options

\begin{tabular}{|c|c|c|c|}
\hline Histologic subtype & Relative chemosensitivity & Primary drug(s) of choice & Substitution drug(s) of choice \\
\hline \multicolumn{4}{|l|}{ Liposarcoma } \\
\hline Myxoid / round-cell liposarcoma & Chemosensitive & DXR & $\begin{array}{l}\text { Trabectedin, IFM/IFM + DXR, } \\
\text { eribulin }\end{array}$ \\
\hline Pleomorphic liposarcoma & Moderately chemosensitive & DXR & $\begin{array}{l}\text { IFM, eribulin, trabectedin, } \text { GEM }^{a} \text {, } \\
\text { DTIC }^{\text {a }}\end{array}$ \\
\hline Dedifferentiated liposarcoma & Moderately chemosensitive & DXR & IFM, eribulin, trabectedin \\
\hline Well-differentiated liposarcoma & Chemoresistant & - & - \\
\hline \multicolumn{4}{|l|}{ Leiomyosarcoma } \\
\hline Uterine leiomyosarcoma & \multirow[t]{2}{*}{ Moderately chemosensitive } & \multirow[t]{2}{*}{ DXR } & \multirow{2}{*}{$\begin{array}{l}\text { GEM }^{\mathrm{a}}+\mathrm{DTX}^{\mathrm{a}} \text {, IFM, eribulin, } \\
\text { pazopanib, trabectedin }\end{array}$} \\
\hline Non-uterine leiomyosarcoma & & & \\
\hline \multicolumn{4}{|l|}{ Other types } \\
\hline Rhabdomyosarcoma & \multirow{2}{*}{$\begin{array}{l}\text { Chemotherapy integral to } \\
\text { management }\end{array}$} & \multicolumn{2}{|l|}{ VAC or VAI/VIE regimen } \\
\hline Ewing sarcoma & & \multicolumn{2}{|l|}{ VDC/IE or VAIA regimen } \\
\hline Synovial sarcoma & Chemosensitive & IFM, DXR & $\begin{array}{l}\text { DTIC }{ }^{a} \text {, eribulin, pazopanib, } \\
\text { trabectedin }\end{array}$ \\
\hline Epithelioid sarcoma & \multirow{4}{*}{ Moderately chemosensitive } & DXR & $\begin{array}{l}\text { IFM, eribulin, pazopanib, } \\
\text { trabectedin }\end{array}$ \\
\hline Myxofibrosarcoma & & DXR & $\begin{array}{l}\text { DTIC , eribulin, pazopanib, } \\
\text { trabectedin }\end{array}$ \\
\hline Fibrosarcoma & & DXR & $\begin{array}{l}\text { DTIC }^{\text {a }}, \text { GEM }^{\text {a }} \text {, eribulin, pazopanib, } \\
\text { trabectedin }\end{array}$ \\
\hline Angiosarcoma & & DXR, PTX & $\begin{array}{l}\text { IFM, GEM }{ }^{\mathrm{a}} \text {, eribulin, trabectedin, } \\
\text { pazopanib }\end{array}$ \\
\hline Alveolar soft part sarcoma & \multirow{3}{*}{ Chemoresistant } & Sunitinib $^{\mathrm{a}}$ & Pazopanib \\
\hline Clear cell sarcoma & & \multirow{2}{*}{-} & \multirow{2}{*}{-} \\
\hline Extraskeletal myxoid chondrosarcoma & & & \\
\hline
\end{tabular}

$D T I C$ dacarbazine, $D T X$ docetaxel, $D X R$ doxorubicin, GEM gemcitabine, IFM ifosfamide, $P T X$ paclitaxel, $V A C$ vincristine, actinomycin $\mathrm{D}$, and cyclophosphamide, $V A I$ vincristine, actinomycin $\mathrm{D}$, and ifosfamide, $V A I A$ vincristine, doxorubicin, ifosfamide, and actinomycin $\mathrm{D}, V D C / I E$ vincristine, doxorubicin, cyclophosphamide, and ifosfamide and etoposide, VIE vincristine, ifosfamide, and etoposide

${ }^{a}$ Not approved for the treatment of STS in Japan

dacarbazine (HR 0.55; 95\% CI 0.42-0.73). This finding suggests that trabectedin may be useful in improving PFS.
In the phase III study of eribulin [18], patients with leiomyosarcoma made up the greatest proportion $(65.7 \%)$ of the study 


\section{Liposarcoma}

\section{1st-line}

\section{Post-DXR}

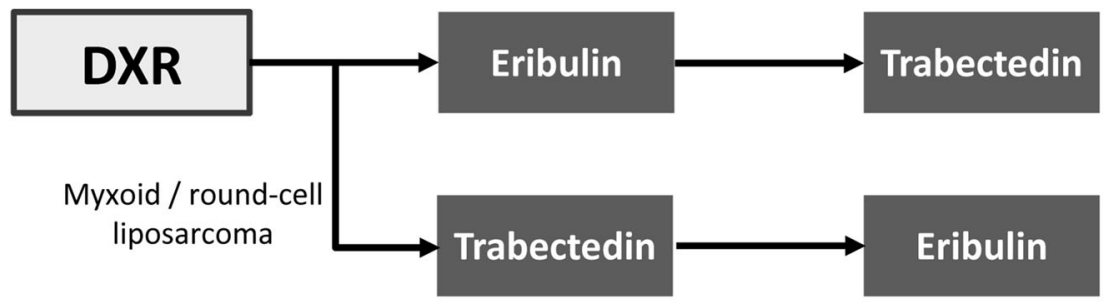

\section{Other options:}

- Other active agent (GEM ${ }^{a}$, DTICa, etc.)

- Pazopanib (note: the phase III study did not include patients with liposarcoma)

- Trabectedin (trabectedin reduced the risk of progressive disease or death in patients with advanced translocation-related sarcoma, including myxoid / roundcell liposarcoma, after standard chemotherapy such as DXR, and is a treatment option for translocation-related sarcoma for the purpose of tumor shrinkage)

- Clinical trial

\section{Leiomyosarcoma}

\section{1st-line}

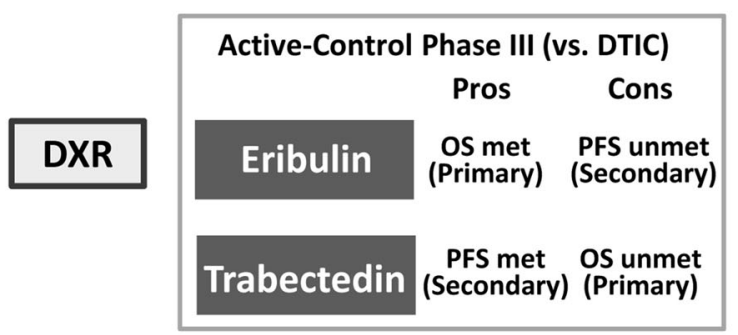

\section{Post-DXR}

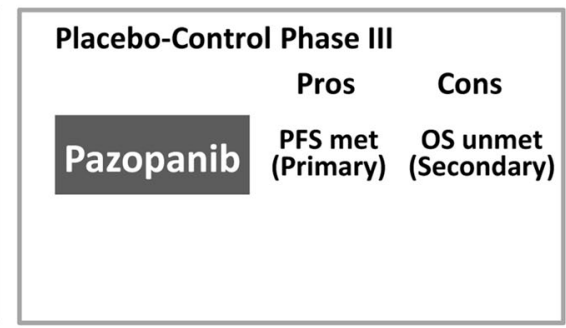

\section{Other options:}

- Other active agent (GEM ${ }^{\mathrm{a}}$, DTICa, GEM ${ }^{\mathrm{a}}+\mathrm{DTX} \mathrm{X}^{\mathrm{a}}$, etc.)

- Clinical trial

\section{Notes}

1. When administering pazopanib and trabectedin, it is necessary to consider the risk of cardiac dysfunction, for example in patients previously treated with anthracycline-containing regimen(s).

2. Ifosfamide is a second-line or later treatment option for liposarcoma and leiomyosarcoma. Patients receiving treatment with ifosfamide require monitoring for renal dysfunction.

3. When administering each drug, it is necessary to consider the PS of patient, adequate shared decision making, and safety issues (see also Table 3).

Fig. 1 Treatment strategies for advanced STS in clinical practice. ${ }^{a}$ Not approved for the treatment of STS in Japan. DTIC dacarbazine, $D X R$ doxorubicin, GEM gemcitabine, DTX docetaxel 
population, and the primary end point of OS was met for the overall population. Subgroup analysis by histologic subtype of the data for OS showed the effect of eribulin on leiomyosarcoma to be similar to that of dacarbazine (HR 0.93; 95\% CI 0.71-1.20). This is thought to be because dacarbazine, known to be effective for patients with leiomyosarcoma [27], was the active comparator. Therefore, eribulin is deemed to be similarly effective to dacarbazine for patients with leiomyosarcoma. Our proposed strategy for the treatment of leiomyosarcoma based on the above information is shown in Fig. 1.

\section{Safety Considerations}

The selection of patients for treatment with any anticancer drug is based not only on considerations of efficacy but also on considerations of safety of the drug. Safety information from preapproval clinical studies is based on safety data from the limited patients selected in accordance with the inclusion criteria. However, in clinical practice, STS patients have more varied baseline characteristics, and some have poor performance status and decreased organ function. Therefore, the information obtained from clinical studies should be supplemented by expert opinions from specialists with appropriate clinical experience.

In this section, we review the results of the safety analyses from the phase III studies of pazopanib, trabectedin, and eribulin [16-18]; a subgroup analysis of Japanese patients in the phase III study of pazopanib [28]; Japanese phase II studies of trabectedin and eribulin $[22,29]$; and the Japanese package insert for each drug [30-32]. We summarize the safety precautions that should be considered when selecting patients for treatment with these drugs in clinical practice, taking into account the administration methods for each drug and our combined clinical experience.

The main safety issues to be considered when selecting patients for treatment with pazopanib, trabectedin, and eribulin are summarized in Table 3. The safety profile of each drug should be considered in addition to its efficacy, and appropriate follow-up should be carried out after administration. Moreover, apart from the adverse events listed here, any potential safety issues should be addressed carefully, and treatment should be individualized according to the clinical condition of each patient.

\section{Pazopanib}

In both the international phase III study of pazopanib [16] and the associated subgroup analysis of data from Japanese patients [28], liver dysfunction was reported as an adverse event. In the pazopanib arm of the overall population $(n=240)$ and in the Japanese subgroup $(n=31)$, the incidence of increased alanine aminotransferase (ALT) (all grades) was $46 \%$ and 52\%, respectively; the incidence of increased aspartate aminotransferase (AST) was 51\% and 65\%, respectively; and the incidence of increased total bilirubin was $29 \%$ and $42 \%$, respectively [28]. Therefore, liver function should be monitored before and during treatment.

In the phase III study, pneumothorax occurred in $3 \%$ of patients $(8 / 240)$ in the pazopanib arm [16], but there were no occurrences in the Japanese subgroup [28]. However, in a post-marketing report based on data from Japanese patients, the prevalence of pneumothorax was reported to be $10.3 \%$ (6/58 patients) [33]; therefore, physicians should be aware of the possible onset or worsening of pneumothorax, particularly in patients with lung metastases.

Because the mechanism of action of pazopanib includes antiangiogenic effects [34], hemorrhage is possible in patients with brain metastases. Therefore, safety information should be provided and explained to patients before administration of this drug.

The Japanese package insert for pazopanib states that patients with risk factors for cardiac dysfunction may have exacerbation of symptoms [30]. Anthracyclines such as doxorubicin, which have cardiotoxic effects, may be used as first-line treatment for advanced STS; thus, caution is required when pazopanib is used as the second-line treatment.

In the phase III study, hypertension (grade 3 or higher) occurred in the pazopanib arm, in both the overall population (grade 3, 7\%; 
Table 3 Major safety issues to be considered when selecting patients for treatment with pazopanib, trabectedin, and eribulin, based on expert opinion

\begin{tabular}{|c|c|c|c|}
\hline & Pazopanib & Trabectedin & Eribulin \\
\hline $\begin{array}{l}\text { Patient background } \\
\text { factors that require } \\
\text { special caution } \\
\text { before } \\
\text { administration }\end{array}$ & $\begin{array}{l}\text { - Liver dysfunction } \\
\text { - Hypertension } \\
\text { - Risk factors for cardiac dysfunction } \\
\text { (caution is particularly needed in } \\
\text { patients who have received previous } \\
\text { treatment with anthracyclines) } \\
\text { - Lung metastases (possibility of onset } \\
\text { or worsening of pneumothorax) } \\
\text { - Brain metastases (possibility of } \\
\text { hemorrhage) }\end{array}$ & $\begin{array}{l}\text { - Bone marrow suppression } \\
\text { - Complication with infectious disease } \\
\text { (possibility of worsening of infection } \\
\text { as a result of bone marrow } \\
\text { suppression) } \\
\text { - Liver dysfunction } \\
\text { - History of treatment with anthracycline } \\
\text { drugs or cardiac dysfunction } \\
\text { (possibility of onset or worsening of } \\
\text { cardiac dysfunction) }\end{array}$ & $\begin{array}{l}\text { - Bone marrow suppression } \\
\text { (contraindicated in patients with severe } \\
\text { bone marrow suppression) } \\
\text { - Liver dysfunction }\end{array}$ \\
\hline \multicolumn{4}{|c|}{ Adverse events that may occur after administration } \\
\hline $\begin{array}{l}\text { Can be self-detected } \\
\text { by patients }\end{array}$ & $\begin{array}{l}\text { - Diarrhea } \\
\text { - Fatigue }\end{array}$ & Fatigue & - \\
\hline $\begin{array}{l}\text { Characteristic to } \\
\text { each drug }\end{array}$ & $\begin{array}{l}\text { - Hair hypopigmentation (patients must } \\
\text { be informed) } \\
\text { - Pneumothorax (physicians should be } \\
\text { aware of the possible onset or } \\
\text { worsening) }\end{array}$ & $\begin{array}{l}\text { - Injection site reaction (as this drug } \\
\text { needs to be administered through a } \\
\text { central venous line, caution is required } \\
\text { for extravasation) } \\
\text { - Rhabdomyolysis (in addition to the } \\
\text { initial symptoms, changes in creatine } \\
\text { phosphokinase concentration should be } \\
\text { monitored) } \\
\text { - Neutropenia/leukopenia }\end{array}$ & $\begin{array}{l}\text { - Peripheral sensory neuropathy (most } \\
\text { event is grade } 1-2) \\
\text { - Neutropenia/leukopenia }\end{array}$ \\
\hline $\begin{array}{l}\text { Route of } \\
\text { administration }\end{array}$ & $\begin{array}{l}\text { - Oral administration ( } 800 \mathrm{mg} \text { once } \\
\text { daily) } \\
\text { - Can be administered to outpatients }\end{array}$ & $\begin{array}{l}\text { - Intravenous infusion (administer at } \\
1.2 \mathrm{mg} / \mathrm{m}^{2} \text { body surface area as a } 24-\mathrm{h} \\
\text { intravenous infusion, every } 21 \text { days, } \\
\text { through a central venous line) } \\
\text { - Requires hospitalization }\end{array}$ & $\begin{array}{l}\text { - Intravenous infusion (administer } \\
1.4 \mathrm{mg} / \mathrm{m}^{2} \text { intravenously over } 2-5 \mathrm{~min} \\
\text { on days } 1 \text { and } 8 \text { of a } 21 \text {-day cycle) } \\
\text { - Can be administered to outpatients }\end{array}$ \\
\hline
\end{tabular}

a Based on expert opinion; information was obtained from the package inserts of each drug (sections such as warnings, contraindications, and careful administration)

grade $4,0 \%$ and the Japanese subgroup (grade 3, 16\%; grade 4, 0\%) [28]. However, protocols for the management of hypertension have been established; thus, control of associated symptoms should be possible in patients who experience hypertension as an adverse effect of pazopanib. Safety information should be provided to patients before administration of the drug, and appropriate monitoring of blood pressure during treatment is essential.

In the pazopanib arm of the phase III study, grade 3 or higher fatigue was reported in less 
than $14 \%$ and in $3 \%$ of patients in the overall population and the Japanese subgroup, respectively; and grade 3 or higher diarrhea in 5\% and $6 \%$, respectively [28]. These adverse effects are readily recognized by patients, but the symptoms are difficult to control. No management of fatigue has been established, and it may become necessary to reduce the dosage of pazopanib in patients experiencing this adverse effect. Regarding diarrhea, if symptoms fail to improve with supportive care, interruption or reduction of dosage is necessary.

In regard to the administration of pazopanib, the oral route is convenient for patients.

\section{Trabectedin}

Abnormal changes in laboratory test values associated with bone marrow suppression were recorded in the clinical studies of trabectedin. In the trabectedin arm $(n=340)$ of the phase III study, neutropenia (grade 3, 21\%; grade 4, 16\%) and anemia (grade $3,14 \%$; grade $4,0 \%$ ) were reported [17]. In the trabectedin $\operatorname{arm}(n=36)$ of the Japanese phase II study, neutropenia (grade 3, 31\%; grade 4, 36\%), leukopenia (grade 3, 31\%; grade 4, 25\%), thrombocytopenia (grade 3, 11\%; grade 4,6\%), lymphopenia (grade 3, 19\%; grade 4, 3\%), and anemia (grade 3, 19\%; grade 4, 0\%) were reported [22]. Therefore, blood tests and careful examination of patients for the presence of infection should be carried out before administration of trabectedin; regular blood tests are also required during treatment.

Febrile neutropenia (grade 3, 11\%; grade 4, $3 \%)$ was reported in the trabectedin arm of the Japanese phase II study [22].

Abnormal changes in laboratory test values associated with liver dysfunction were also recorded in the clinical studies of trabectedin. In the trabectedin arm of the phase III study, increased ALT (grade 3, 25\%; grade 4, 1\%), increased AST (grade 3, 12\%; grade 4, 1\%), and increased alkaline phosphatase (ALP) (grade 3, $1 \%$; grade $4,0 \%)$ were reported [17]. In the trabectedin arm of the Japanese phase II study, increased ALT (grade 3, 47\%; grade 4, 14\%), increased AST (grade 3, 33\%; grade 4, 8\%), increased gamma-glutamyltransferase (grade 3 , $22 \%$; grade $4,3 \%$ ), and increased ALP (grade 3 ,
$6 \%$; grade $4,0 \%)$ were reported [22]. In the same study, grade 1 or 2 increased blood bilirubin (11\%) was also reported in the trabectedin arm [22]. In clinical practice, we have encountered serious cases of liver dysfunction in patients treated with trabectedin. Therefore, monitoring of liver function is required in these patients, both before and during treatment.

As with pazopanib, trabectedin may also cause the onset or worsening of cardiac dysfunction when administered to patients with a history of treatment with anthracycline drugs and those with cardiac dysfunction. Before administration of trabectedin, patients are likely to have a history of treatment with doxorubicin, which has cardiotoxic effects; therefore particular caution is required.

Rhabdomyolysis (grade 4, 3\%) and increased creatine phosphokinase (grade $3,6 \%$; grade 4 , $0 \%)$ were reported in the trabectedin arm of the Japanese phase II study [22], and we have encountered serious cases in trabectedin-treated patients in clinical practice. In addition to vigilance for the initial symptoms (e.g., muscular pain and weakness), monitoring is required for increased creatine phosphokinase or increased blood or urinary myoglobin concentration. Appropriate management, including discontinuation of treatment, should be taken if patients had abnormal changes in these values, and physicians and medical staff should be aware of this before the start of treatment.

Fatigue of grade 3 or higher was reported in $6 \%$ and $3 \%$ of patients in the trabectedin arm of the phase III study and the Japanese phase II study, respectively.

Because trabectedin is administered by $24-\mathrm{h}$ intravenous infusion using a central venous catheter or port, hospitalization is required. Tissue necrosis may occur, and extravasation is a concern. If extravasation occurs, the administration of trabectedin should be interrupted immediately; medical staff should be aware of this measure.

\section{Eribulin}

Abnormal changes in laboratory test values associated with bone marrow suppression were recorded in the clinical studies of eribulin. In the phase III study (eribulin arm, $n=226$ ), 
neutropenia (grade 3, 20\%; grade 4, 15\%), leukopenia (grade 3, 8\%; grade 4, 2\%), and thrombocytopenia (grade $3,<1 \%$; grade $4,0 \%$ ) were reported [18]. In the Japanese phase II study $(n=51)$, neutropenia (grade $\geq 3,86 \%)$, leukopenia (grade $\geq 3,75 \%$ ), lymphopenia (grade $\geq 3,33 \%$ ), anemia (grade $\geq 3,14 \%$ ), and febrile neutropenia (grade $\geq 3,8 \%$ ) were reported [29]. The incidence of adverse events associated with bone marrow suppression reported in the clinical studies of eribulin was lower than in those of trabectedin $[17,22]$, although direct comparison of safety data is difficult because of the different background characteristics of the patients in these studies. However, close examination for signs of bone marrow suppression is still required before administration of eribulin, as well as appropriate monitoring after administration, because bone marrow suppression can affect vital functions. The incidence of febrile neutropenia was higher in the Japanese phase II study [29] than the phase III study [18]. However, the overall incidence of febrile neutropenia was less than $10 \%$ in both studies.

Abnormal changes in laboratory test values associated with liver dysfunction were recorded in the Japanese phase II study, namely ALT increase (grade $\geq 3,6 \%$ ) and AST increase (grade $\geq 3,4 \%$ ) [29]. In clinical practice, we have not encountered any serious cases of liver dysfunction in patients treated with eribulin; however, it is essential to assess liver function before its administration.

In the phase III study of eribulin, grade 3 or higher peripheral sensory neuropathy (grade 3 , $2 \%$; grade $4,0 \%$ ) was recorded in the eribulin arm [18]. In contrast, in the Japanese phase II study, the severity of peripheral sensory neuropathy was grade 1 and 2 [29]. The likelihood of the onset of peripheral sensory neuropathy depends largely on previous treatment; therefore, caution is required in patients previously treated with drugs such as vincristine that cause peripheral sensory neuropathy. However, vincristine is generally administered to young children with rhabdomyosarcoma; therefore, its target patients differ from those of eribulin. However, as peripheral sensory neuropathy may occur as a result of cumulative toxicity, caution is required in the long-term administration of eribulin.
Overall, we consider that the toxicities of eribulin are manageable, and that management of adverse effects of eribulin is relatively straightforward on the basis of our experience.

Eribulin needs to be administered by intravenous infusion. However, as this takes only 2-5 $\mathrm{min}$, it is possible to administer the drug in an outpatient visit.

\section{DISCUSSION}

In light of new treatment options that have been developed with the aim of improving OS in patients with advanced STS, we propose in this article an optimal treatment approach to the use of pazopanib, trabectedin, and eribulin based on current evidence from the relevant clinical studies. In addition, regarding the safety profiles of each drug, the review of clinical study data and our experience in clinical practice may be useful for the selection of appropriate patients for treatment with these drugs. The primary end points of pivotal studies were used in this article. However, because the primary end point of trabectedin and eribulin was OS, and that of pazopanib was PFS, the results of these studies were not directly comparable. For this reason, in this article we have described, in an objective manner, the characteristics of these drugs.

In Japan, pazopanib, trabectedin, and eribulin can be used for all histologic subtypes of STS. However, each drug has a different profile. For the proper use of these drugs, and to maximize their benefit to patients, it is necessary to understand the efficacy and safety profiles of each drug, and to provide treatment in accordance with evidence-based medicine. To achieve these objectives in clinical practice, first, anticancer drugs should be selected according to their sensitivity to histologic subtypes and safety profile on the basis of the findings of clinical trials, as described in this article. In addition, consideration of expert opinions based on clinical experience is recommended. For instance, when choosing an anticancer drug for second-line treatment of liposarcoma and leiomyosarcoma, in addition to the clinical evidence, safety issues such as the 
onset or worsening of cardiac dysfunction caused by doxorubicin should be considered. Furthermore, retrospective studies from Japan have shown the inferior efficacy of pazopanib in the treatment of liposarcoma compared with other subtypes [35, 36]. Ifosfamide can also be an option for second-line and later treatment for metastatic STS, especially in cases of fibrosarcoma and synovial sarcoma. However, the response rate for leiomyosarcoma has been shown to be lower than for fibrosarcoma and synovial sarcoma [37-39]. In addition, this treatment requires hospitalization, and attention should be paid to the possibility of renal dysfunction. The efficacy of gemcitabine, given alone or in combination with other drugs, has been studied. In a randomized phase II study in patients with metastatic soft tissue sarcoma, the combination of gemcitabine and docetaxel resulted in superior PFS and OS compared with gemcitabine alone, but with increased toxicity [40]. However, the combination regimens of gemcitabine with dacarbazine or vinorelbine have not been approved in Japan.

For other histologic subtypes, pazopanib may be effective for synovial sarcoma on the basis of the results of the subgroup analysis of the international phase III study, although the number of patients was limited $(n=30)$ [16]. Predictive analysis, using Cox models, of PFS according to histologic subtype also showed the effectiveness of pazopanib against synovial sarcoma, with no significant interaction between histologic subtypes [16]. The number of STS patients with histologic subtypes other than liposarcoma and leiomyosarcoma is small, and it is therefore difficult to evaluate the efficacy of each drug in clinical studies. The phase III studies of trabectedin and eribulin included patients with leiomyosarcoma and liposarcoma but not undifferentiated pleomorphic sarcoma $[17,18]$. In contrast, the phase III study of pazopanib included patients with undifferentiated pleomorphic sarcoma [16]. Therefore, it is inappropriate to discuss in this article the treatment of undifferentiated pleomorphic sarcomas. The phase II studies of pazopanib, trabectedin, and eribulin included patients with undifferentiated pleomorphic sarcoma, but the data are insufficient to enable us to make specific recommendations for treatment $[20,29,41,42]$. A more practical approach to evaluation of the effectiveness of pazopanib, trabectedin, and eribulin against specific histologic subtypes of STS is recommended, namely to generate evidence by obtaining clinical data retrospectively. For example, the Japanese Musculoskeletal Oncology Group have conducted a study to evaluate the effects of pazopanib in Japanese STS patients, based on safety data gathered by post-marketing surveillance and efficacy data obtained at the study group sites [35]. It is expected to accumulate realworld data including information on various histologic subtypes.

To provide more effective treatment, it is preferable to identify biomarkers. Trabectedin has been shown to reduce the risk of progressive disease or death in patients with advanced translocation-related sarcoma [22]. In addition, tumor biomarkers for safety and efficacy end points have been incorporated into the phase III study of eribulin as a planned exploratory analysis [18]; the results are awaited with interest.

It may be necessary in future clinical studies to evaluate sequential therapy, for example the efficacy in terms of OS of trabectedin after eribulin or OS of eribulin after trabectedin in the treatment of liposarcoma. In a phase III study, eribulin monotherapy resulted in no significant difference in PFS but significantly improved OS compared with dacarbazine, suggesting that eribulin might have induced biological effects on tumor vascularization, microenvironment, and metastasis [18].

We have proposed treatment strategies for advanced STS by histologic subtype as well as safety considerations based on our clinical experience in the use of pazopanib, trabectedin, and eribulin. These would benefit patients by maximizing the therapeutic effects and enhancing the proper use of these drugs.

\section{ACKNOWLEDGEMENTS}

Sponsorship for this article, including processing charges, was funded by Eisai Co., Ltd (Tokyo, Japan). 
All named authors meet the International Committee of Medical Journal Editors (ICMJE) criteria for authorship for this manuscript, take responsibility for the integrity of the work as a whole, and have given final approval for the version to be published. All authors had full access to all of the data in this study and take complete responsibility for the integrity of the data and accuracy of the data analysis.

Medical writing assistance was provided by Hiroki Matsushita of ShiftZero K.K. (Tokyo, Japan) and editorial assistance was provided by Rie Ishibashi on behalf of inScience Communications, Springer Healthcare (Tokyo, Japan). Support for these assistances was funded by Eisai Co., Ltd.

Disclosures. Akira Kawai has received honoraria for serving as an advisory board member for Novartis Pharma K.K., Taiho Pharmaceutical Co., Ltd., Eisai Co., Ltd., and Eli Lilly Japan K.K. Kan Yonemori has received speakers' honoraria from Eisai Co., Ltd., Taiho Pharmaceutical Co., Ltd., and Novartis Pharma K.K. Shunji Takahashi has received research funding and speakers' honoraria from Taiho Pharmaceutical Co., Ltd., Eisai Co., Ltd., and Novartis Pharma K.K. Nobuhito Araki has received speakers' honoraria and research funding from Taiho Pharmaceutical Co., Ltd. Takafumi Ueda serves as a chief organizer of the Japanese Musculoskeletal Oncology Group and received research funding from Novartis Pharma K.K., received honoraria for serving as speaker and advisory board member for Taiho Pharmaceutical Co., Ltd., and received research funding and honoraria for serving as an advisory board member and a speaker from Eisai Co., Ltd.

Compliance with Ethics Guidelines. This article is based on previously conducted studies and does not involve any new studies of human or animal subjects performed by any of the authors.

Data Availability. Data sharing is not applicable to this article, because no datasets were generated or analyzed in the course of our research.
Open Access. This article is distributed under the terms of the Creative Commons Attribution-NonCommercial 4.0 International License (http://creativecommons.org/licenses/ by-nc/4.0/), which permits any noncommercial use, distribution, and reproduction in any medium, provided you give appropriate credit to the original author(s) and the source, provide a link to the Creative Commons license, and indicate if changes were made.

\section{REFERENCES}

1. Ducimetière $F$, Lurkin $A$, Ranchère-Vince $D$, et al. Incidence of sarcoma histotypes and molecular subtypes in a prospective epidemiological study with central pathology review and molecular testing. PLoS One. 2011;6(8):e20294.

2. Toro JR, Travis LB, Wu HJ, Zhu K, Fletcher CDM, Devesa SS. Incidence patterns of soft tissue sarcomas, regardless of primary site, in the Surveillance, Epidemiology and End Results program, 1978-2001: an analysis of 26,758 cases. Int J Cancer. 2006;119:2922-30.

3. Siegel RL, Miller KD, Jemal A. Cancer statistics, 2017. CA Cancer J Clin. 2017;67:7-30.

4. Japanese Orthopaedic Association Committee on Musculoskeletal Tumor. Soft tissue tumor registry in Japan 2015. Tokyo: National Cancer Center; 2015.

5. Schöffski P, Cornillie J, Wozniak A, Li H, Hompes D. Soft tissue sarcoma: an update on systemic treatment options for patients with advanced disease. Oncol Res Treat. 2014;37:355-62.

6. Fletcher CDM, Bridge JA, Hogendoorn PCW, Mertens $\mathrm{F}$, editors. WHO classification of tumours of soft tissue and bone. 4th ed. Lyon: IARC; 2013.

7. von Mehren M, Randall RL, Benjamin RS, et al. Soft tissue sarcoma, version 2.2016, NCCN clinical practice guidelines in oncology. J Natl Compr Canc Netw. 2016;14:758-86.

8. Stiller CA, Trama A, Serraino D, et al. RARECARE Working Group. Descriptive epidemiology of sarcomas in Europe: report from the RARECARE project. Eur J Cancer. 2013;49:684-95.

9. Elias A, Ryan L, Sulkes A, Collins J, Aisner J, Antman $\mathrm{KH}$. Response to mesna, doxorubicin, ifosfamide, and dacarbazine in 108 patients with metastatic or 
unresectable sarcoma and no prior chemotherapy. J Clin Oncol. 1989;7:1208-16.

10. Antman K, Crowley J, Balcerzak SP, et al. An intergroup phase III randomized study of doxorubicin and dacarbazine with or without ifosfamide and mesna in advanced soft tissue and bone sarcomas. J Clin Oncol. 1993;11:1276-85.

11. Edmonson JH, Ryan LM, Blum RH, et al. Randomized comparison of doxorubicin alone versus ifosfamide plus doxorubicin or mitomycin, doxorubicin, and cisplatin against advanced soft tissue sarcomas. J Clin Oncol. 1993;11:1269-75.

12. Santoro A, Tursz T, Mouridsen H, et al. Doxorubicin versus CYVADIC versus doxorubicin plus ifosfamide in first-line treatment of advanced soft tissue sarcomas: a randomized study of the European Organization for Research and Treatment of Cancer Soft Tissue and Bone Sarcoma Group. J Clin Oncol. 1995;13:1537-45.

13. Judson I, Verweij J, Gelderblom H, et al. Doxorubicin alone versus intensified doxorubicin plus ifosfamide for first-line treatment of advanced or metastatic soft-tissue sarcoma: a randomised controlled phase 3 trial. Lancet Oncol. 2014;15:415-23.

14. Martin-Broto J, Pousa AL, de Las Peñas R, et al. Randomized phase II study of trabectedin and doxorubicin compared with doxorubicin alone as first-line treatment in patients with advanced soft tissue sarcomas: a Spanish Group for Research on Sarcoma study. J Clin Oncol. 2016;34:2294-302.

15. ESMO/European Sarcoma Network Working Group. Soft tissue and visceral sarcomas: ESMO Clinical Practice Guidelines for diagnosis, treatment and follow-up. Ann Oncol. 2014;25:iii102-12.

16. van der Graaf WTA, Blay JY, Chawla SP, et al. Pazopanib for metastatic soft-tissue sarcoma (PALETTE): a randomised, double-blind, placebo-controlled phase 3 trial. Lancet. 2012;379:1879-86.

17. Demetri GD, von Mehren M, Jones RL, et al. Efficacy and safety of trabectedin or dacarbazine for metastatic liposarcoma or leiomyosarcoma after failure of conventional chemotherapy: results of a phase III randomized multicenter clinical trial. J Clin Oncol. 2016;34:786-93.

18. Schöffski P, Chawla S, Maki RG, et al. Eribulin versus dacarbazine in previously treated patients with advanced liposarcoma or leiomyosarcoma: a randomised, open-label, multicentre, phase 3 trial. Lancet. 2016;387:1629-37.

19. Eli Lilly. A study of doxorubicin plus olaratumab (LY3012207) in participants with advanced or metastatic soft tissue sarcoma (ANNOUNCE).
https://clinicaltrials.gov/ct2/show/NCT02451943. Accessed 11 Dec 2016.

20. Sleijfer S, Ray-Coquard I, Papai Z, et al. Pazopanib, a multikinase angiogenesis inhibitor, in patients with relapsed or refractory advanced soft tissue sarcoma: a phase II study from the European Organisation for Research and Treatment of Cancer-Soft Tissue and Bone Sarcoma Group (EORTC study 62043). J Clin Oncol. 2009;27:3126-32.

21. Ueda T, Kakunaga S, Ando M, et al. Phase I and pharmacokinetic study of trabectedin, a DNA minor groove binder, administered as a 24 -h continuous infusion in Japanese patients with soft tissue sarcoma. Invest New Drugs. 2014;32:691-9.

22. Kawai A, Araki N, Sugiura H, et al. Trabectedin monotherapy after standard chemotherapy versus best supportive care in patients with advanced, translocation-related sarcoma: a randomised, open-label, phase 2 study. Lancet Oncol. 2015;16:406-16.

23. Grimer R, Judson I, Peake D, Seddon B. Guidelines for the management of soft tissue sarcomas. Sarcoma. 2010;2010:506182.

24. Blay JY, Sleijfer S, Schöffski P, et al. International expert opinion on patient-tailored management of soft tissue sarcomas. Eur J Cancer. 2014;50:679-89.

25. Van Glabbeke M, Verweij J, Judson I, Nielsen OS, EORTC Soft Tissue and Bone Sarcoma Group. Progression-free rate as the principal end-point for phase II trials in soft-tissue sarcomas. Eur J Cancer. 2002;38:543-9.

26. Osgood CL, Chuk MK, Theoret MR, et al. FDA approval summary: eribulin for patients with unresectable or metastatic liposarcoma who have received a prior anthracycline-containing regimen. Clin Cancer Res. 2017. doi:10.1158/1078-0432. CCR-16-2422.

27. García-Del-Muro X, López-Pousa A, Maurel J, et al. Randomized phase II study comparing gemcitabine plus dacarbazine versus dacarbazine alone in patients with previously treated soft tissue sarcoma: a Spanish Group for Research on Sarcomas study. J Clin Oncol. 2011;29:2528-33.

28. Kawai A, Araki N, Hiraga $\mathrm{H}$, et al. A randomized, double-blind, placebo-controlled, phase III study of pazopanib in patients with soft tissue sarcoma: results from the Japanese subgroup. Jpn J Clin Oncol. 2016;46:248-53.

29. Kawai A, Araki N, Naito Y, et al. Phase 2 study of eribulin in patients with previously treated advanced or metastatic soft tissue sarcoma. Jpn J Clin Oncol. 2017;47:137-44. 
30. Japanese package insert for Votrient ${ }^{\circledR}$. 2015. http:// database.japic.or.jp/pdf/newPINS/00060782.pdf. Accessed 11 Dec 2016.

31. Japanese package insert for Yondelis ${ }^{\circledR}$. 2016. http:// database.japic.or.jp/pdf/newPINS/00065945.pdf. Accessed 11 Dec 2016.

32. Japanese package insert for Halaven ${ }^{\circledR}$. 2016. http:// database.japic.or.jp/pdf/newPINS/00059691.pdf. Accessed 11 Dec 2016.

33. Nakano K, Motoi N, Tomomatsu J, et al. Risk factors for pneumothorax in advanced and/or metastatic soft tissue sarcoma patients during pazopanib treatment: a single-institute analysis. BMC Cancer. 2016;16:750.

34. Kumar R, Knick VB, Rudolph SK, et al. Pharmacokinetic-pharmacodynamic correlation from mouse to human with pazopanib, a multikinase angiogenesis inhibitor with potent antitumor and antiangiogenic activity. Mol Cancer Ther. 2007;6:2012-21.

35. Nakamura T, Matsumine A, Kawai A, et al. The clinical outcome of pazopanib treatment in Japanese patients with relapsed soft tissue sarcoma: a Japanese Musculoskeletal Oncology Group (JMOG) study. Cancer. 2016;122:1408-16.

36. Nakano K, Motoi N, Inagaki L, et al. Differences in the responses to pazopanib and the prognoses of soft tissue sarcomas by their histological eligibility for the PALETTE study. Jpn J Clin Oncol. 2015;45:449-55.
37. Antman KH, Ryan L, Elias A, Sherman D, Grier HE. Response to ifosfamide and mesna: 124 previously treated patients with metastatic or unresectable sarcoma. J Clin Oncol. 1989;7:126-31.

38. Le Cesne A, Antoine E, Spielmann M, et al. Highdose ifosfamide: circumvention of resistance to standard-dose ifosfamide in advanced soft tissue sarcomas. J Clin Oncol. 1995;13:1600-8.

39. Patel SR, Vadhan-Raj S, Papadopolous N, et al. High-dose ifosfamide in bone and soft tissue sarcomas: results of phase II and pilot studiesdose-response and schedule dependence. J Clin Oncol. 1997;15:2378-84.

40. Maki RG, Wathen JK, Patel SR, et al. Randomized phase II study of gemcitabine and docetaxel compared with gemcitabine alone in patients with metastatic soft tissue sarcomas: results of Sarcoma Alliance for Research Through Collaboration study 002 [corrected]. J Clin Oncol. 2007;25:2755-63.

41. De Sanctis R, Marrari A, Marchetti S, et al. Efficacy of trabectedin in advanced soft tissue sarcoma: beyond lipo- and leiomyosarcoma. Drug Des Devel Ther. 2015;9:5785-91.

42. Schöffski P, Ray-Coquard IL, Cioffi A, et al. Activity of eribulin mesylate in patients with soft-tissue sarcoma: a phase 2 study in four independent histological subtypes. Lancet Oncol. 2011;12:1045-52. 